\title{
Optimal Resource Allocation and VCG Auction-Based Pricing for H.264 Scalable Video Quality Maximization in $4 \mathrm{G}$ Wireless Systems
}

\author{
Shreyans Parakh and Aditya K. Jagannatham \\ Department of Electrical Engineering, Indian Institute of Technology, Kanpur 208016, India \\ Correspondence should be addressed to Aditya K. Jagannatham, adityaj@iitk.ac.in
}

Received 15 October 2011; Revised 21 December 2011; Accepted 31 January 2012

Academic Editor: Raouf Hamzaoui

Copyright (C) 2012 S. Parakh and A. K. Jagannatham. This is an open access article distributed under the Creative Commons Attribution License, which permits unrestricted use, distribution, and reproduction in any medium, provided the original work is properly cited.

\begin{abstract}
We present novel schemes for optimal OFDMA bitrate allocation towards video quality maximization in H.264 scalable video coding (SVC)-based 4G wireless systems. We use the rate and quality models for video characterization of the SVC extension of the H.264/AVC and develop the framework for optimal scalable video transmission. Subsequently, we derive the closed form solution of the optimal H.264 scalable video quantization parameter for sum video quality maximization in unicast and multicast 4G WiMAX adaptive modulation and coding (AMC) scenarios. We also formulate a Vickrey-Clarke-Groves (VCG) auctionbased time-frequency (TF) resource pricing scheme for dynamic bitrate allocation and simultaneous prevention of video quality degradation by malicious users for H.264-based scalable video transmission. Simulation results demonstrate that application of the proposed optimal 4G OFDMA schemes for unicast/multicast video quality maximization yield significantly superior performance in comparison to fixed rate video agnostic allocation.
\end{abstract}

\section{Introduction}

The rapid rise in the demand for ubiquitous mobile broadband wireless access has spurred the development of $4 \mathrm{G}$ wireless standards such as LTE and WiMAX. These technologies provide high data rates and reliable wireless services to the users. A significant component of the $4 \mathrm{G}$ wireless traffic comprises of video and multimedia-based rich applications such as surveillance, multimedia streaming, mobile TV, and video conferencing. A typical $4 \mathrm{G}$ wireless communication scenario for the above-described applications is shown in Figure 1. The key challenge in $4 \mathrm{G}$ cellular networks in the context of video transmission is to support reliable video streaming over the erratic fading wireless channels. This fading nature can potentially result in intolerable jitter and latency resulting in poor end-user experience for the highly sensitive multimedia applications. The fading nature of the wireless channel can be successfully mitigated using orthogonal frequency division multiplexing (OFDM) $[1,2]$, thus ensuring inter-symbol interference free transmission across frequency-selective wireless channels. orthogonal frequency division for multiple access (OFDMA) is the multiple access technology based on OFDM in which different users (unicast) or groups of users (multicast) are allocated a fraction of the total subcarriers over a period of time. This is also known as time-frequency resource allocation in OFDMA systems.

Supporting video applications on wireless links necessitates the development of sophisticated multimedia codecs tailored for applicability in the erratic mobile wireless environment. A unique challenge for video transmission in $4 \mathrm{G}$ wireless systems is to ensure quality of video transmission over the time-varying fading wireless channel to mobile users with devices of disparate capabilities and QoS requirements. This has lead to the development of the scalable video coding (SVC) profile of the H.264/AVC $[3,4]$ which can be readily adopted for video transmission in unicast and multicast wireless scenarios. Scalable video coding enables the video content to be coded and stored at its highest fidelity levels, from which partial bit streams of lower fidelity can be extracted dynamically and adapted to meet the requirements 
of the users and the wireless links. The bitrate and video quality of the coded video stream depend on the combination of frame rate, spatial resolution, and quantization parameter [5]. Hence, it is essential to judiciously choose the coded video parameters to maximize the end user video quality and experience. Further, this has a direct impact on the end user quality of service (QoS) aspects such as jitter and latency. Compared to the spatial and temporal modes of scalability, the quantization parameter of a video stream can be adapted on a much finer scale and allows for greater flexibility towards optimal time-frequency resource allocation. The allocated bitrate and quality of video depends critically on the intrinsic video motion parameters. In this context, we consider a framework for optimal H.264-coded video ratebased time-frequency resource allocation at the $4 \mathrm{G}$ wireless Base Station (BS) for video quality maximization. In this paradigm, the users request the videos either individually or in multicast groups, and the server allocates time/frequency resources in the OFDMA system.

Previous works such as in [6] consider scheduling and resource allocation based on priority and latency. However, most such previous approaches are not specialized to the context of video and do not take the scalable nature of video transmission into consideration. This leads to suboptimal resource allocation and a net decrease in the video quality delivered to the end users. The authors in [7] allocate the time/frequency resources for real-time layered video transmission in WiMAX assuming fixed bitrate allocation to each multicast group. The utility of each multicast group is assumed to be a concave function of the bitrate allocated. However, the considered rate dependent generic utility function is not an accurate representation of the video quality. In our work, we consider the true perceptual quality-based utility functions. Hence, our framework provides a better end user video experience since it optimizes the relevant video quality directly. In [8], a scheme is proposed for allocation of the time resources in a HSDPA cellular network. The proposed scheme therein requires users to request a video quality level, with video quality defined as a function of the number of enhancement layers and the cumulative data rate. However, this framework does not consider the dependence of video quality and bitrate on the quantization quality and frame rate. Further, it does not consider a realistic optimization framework as compared to the one illustrated in this work in the context of a practical 4G WiMAX system. Hence, the key to efficient resource allocation in $4 \mathrm{G}$ wireless systems lies in the interpretation of the characteristic video rate and quality parameters which lead to optimal bitrate allocation. This necessitates the development of optimal schemes for time-frequency resource allocation and management. The proposed optimal time-frequency resource allocation scheme computes the bitrate to be allocated to the video sequences in the physical layer for video quality maximization.

Therefore, we consider a framework for optimal OFDMA time-frequency resource allocation based on the characteristic video quality and bitrate models of the scalable video bit streams as functions of quantization parameter and frame rate. We compute the bitrate models of the H.264 SVC coded streams using the JSVM [9] reference codec and employ the standard video parameters from works such as $[5,10]$ to characterize the quality dependence on frame rate, quantization parameter of the coded videos. Based on these models, we formulate a constrained convex optimization problem for optimal OFDMA time-frequency resource allocation. We employ the robust framework of convex optimization [11] to present a closed form expression for computation of the optimal coded video parameters. The server can employ these parameters to compute the optimal resource allocation based on the requirements of the users and availability of the bandwidth. This efficient utilization of the available bandwidth results in maximizing the quality of the transmitted video and end user video experience. Our results demonstrate that optimization using the proposed model yields significant enhancement in the video quality as compared to the video agnostic equal bitrate allocation for unicast/multicast scenarios in the OFDMA system.

Further, in practical $4 \mathrm{G}$ systems, malicious users can distort the resource allocation scheme at the QoS enforcement points (such as base stations and service gateways in WiMAX) by misreporting the parameter values, thereby resulting in suboptimal resource allocation and disproportionate benefits to the malicious users. The optimal solution and the highest video quality is hence obtained only when the parameters are reported accurately by the unicast/multicast subscribers or service providers.

Game theory [12, 13] -based auctioning provides a framework to allocate resources in the presence of such distorting malicious users. This along with the optimization framework can be used to allocate bitrate to video sequences which discourages malicious users. Its applications have been recently extended to the field of wireless communication, especially in the context of resource optimization [14]. The authors in [15] define a utility function based on transmission rate and packet error probability and aim to achieve best quality of experience. In the context of $4 \mathrm{G}$ wireless video communication, game theory-based VickreyClarke-Groves (VCG) auction procedure can be adapted for time-frequency (TF) resource allocation. The auctioned item in this context is the bitrate corresponding to the allotted TF resources, and the bidders/decision makers are the service providers or users themselves. The auctioneer is the QoS policy enforcer in the $4 \mathrm{G}$ wireless network. This interaction between various decision makers is akin to a strategic game, and the decision makers are also termed as players in the nomenclature of game theory. We assume that all the players are rational and are driven towards utility maximization. Each user reports the characteristic video parameter values to the policy enforcer to calculate the sum utility function. Unlike conventional utility-based exclusively on video quality, the VCG procedure employs the pricingbased net utility function, which prices the TF resources in accordance with the allocation. Therefore, knowledge of the characteristic video parameters is critical for optimizing the bitrate and quality of the streamed videos.

Some research regarding the use of game theory with malicious users has been considered in [16] in the context of peer-to-peer live streaming. The research in [17] proposes 


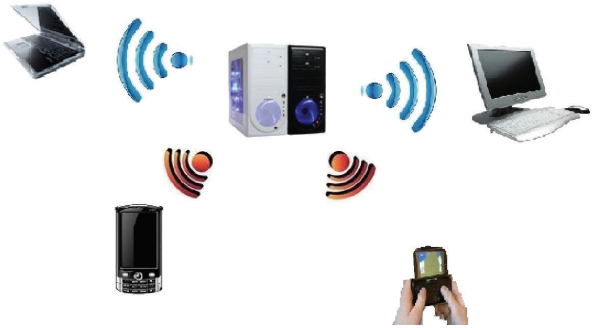

FIgURE 1: A wireless communication scenario.

a Vickrey scheme for computing the shortest path in a decentralized network. The authors in [18] present the application of a VCG procedure in mechanism design. In this paper, we are primarily concerned about misreporting of the quantizer-based rate and quality parameter values. The framework can readily be extended to VCG-based optimization for malicious users misreporting other parameter values.

In the simulation results, we specialize our proposed algorithm taking into account the different modulation and coding rates in a $4 \mathrm{G}$ WiMAX scenario and demonstrate that the proposed optimization scheme provides significant improvement in video quality over the content agnostic nonscalable equal symbol rate allocation scheme for unicast and multicast scenarios. We further consider that the parameters may be subverted to benefit a group of users. The proposed VCG procedure ensures that the users misreporting the parameters are punished by the QoS enforcer through higher resource pricing, in turn resulting in a reduced net utility for the malicious user. Hence, the VCG procedure naturally discourages users' malicious tendency towards misreporting and forces them to report accurate parameter values towards net utility maximization.

The rest of the paper is organized as follows. Section 2 describes the underlying framework for $4 \mathrm{G}$ WiMAX-based H.264 scalable video transmission considered in this paper and the rate and quality models of the videos. Subsequently, in Section 3 we describe the scheme for optimal video TF resource allocation in an OFDM frame. Section 4 describes the VCG procedure-based resource allocation to avoid misreporting of parameters by malicious users. In Section 5 we present the simulation results for the proposed optimal unicast/multicast video resource allocation schemes in 4G OFDMA wireless systems and a performance comparison with the existing schemes. Finally, we conclude the paper in Section 6.

\section{System Model and 4G OFDMA WiMAX Framework}

In OFDMA systems, the high data rate input stream is divided into a multitude of parallel low data rate streams which are subsequently loaded onto the orthogonal subcarriers. Each symbol in the time domain comprises of several orthogonal subcarriers. A few such subcarriers are designated as pilot and guard subcarriers which comprise an overhead in

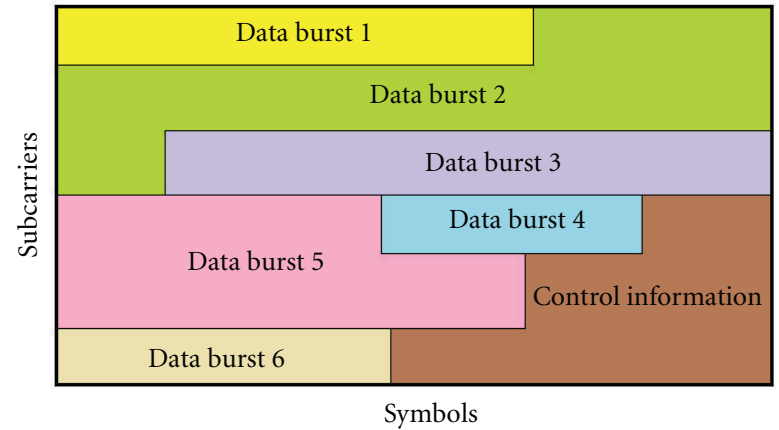

FIgURE 2: Rough schematic of OFDM frame in WiMAX.

the OFDMA system. Pilot subcarriers are employed to estimate the timing and frequency synchronization parameters so that the offset errors are minimized, while the guard subcarriers avoid overlap with adjacent OFDM bands. The OFDMA scheduler allocates the time/frequency resource blocks, which are characterized by the allotted OFDM symbols/subcarriers, respectively, to the users. The bitrate of the OFDMA system depends on the number of symbols in each OFDM frame, the number of subcarriers used in each symbol, the modulation, and channel coding formats employed. Figure 2 presents the rough schematic of an OFDMA frame in WiMAX.

In this context, the $4 \mathrm{G}$ wireless cellular standard WiMAX [19], which employs OFDMA in the physical layer for transmission of bits was designed to provide a high data rate broadband air interface to its users coupled with seamless data transfer under high speed mobility. WiMAX provides services such as unsolicited grants service (UGS) for constant bitrate VOIP applications, real-time polling service (rtPS) for real time applications such as video transmission, non real time polling service for large data transfers and best effort service for web applications. The scheduler present at the base station helps in optimally allocating the bandwidth resources, aimed at avoiding traffic congestion and data starvation. Thus, the DL scheduler has the critical tasks of optimal bandwidth allocation, choosing the modulation and coding schemes and data bursts depending on the service priority and wireless link quality determined from the channel quality indicator (CQICH) feedback channel. It then generates the UL/DL MAP containing the control information for users to access their bursts. Hence, our proposed model aims at optimally allocating the timefrequency resources in the UL and DL scheduler to maximize the net video quality.

2.1. Scalable Video Rate and Quality Models. The parametric models given in [5] can be conveniently employed to model the video bitrate. As proved in this work, we model the rate as a product of the normalized functions of the frame rate $t$ and quantization parameter $q$. We employ the JSVM reference codec to compute the rate parameters for quantization parameter in the range $15 \leq q \leq 40$ with intervals of $q=5$, and frame rates $t=15,30 \mathrm{fps}$. We employed four temporal layers and one quality layer in JSVM to obtain the bitrate for 
these layers. It is to be noted that quantization parameter and quantization step size $\left(q_{s}\right)$ are related as $q=4+6 \log _{2}\left(q_{s}\right)$. The normalized rate functions $R_{t}(t), R_{q}(q)$ of the frame rate $t$ and quantization parameter $q$ respectively are given as

$$
R_{t}(t)=\left(\frac{1-e^{-c t / t_{\max }}}{1-e^{-c}}\right), \quad R_{q}(q)=e^{d\left(1-q / q_{\min }\right)} .
$$

The video characteristic parameters $c$ and $d$ model the bitrate variation as a function of the frame rate and quantization parameter, respectively. The parameters $c$ and $d$ are higher for videos with low motion content. These video characteristic parameters $c$ and $d$ are obtained by minimizing the mean squared-error (MSE) between the measured rate obtained using the JSVM codec and the modeled video sequences for frame rates $15 \mathrm{fps}$ and $30 \mathrm{fps}$. Frame rates lower than $15 \mathrm{fps}$ result in noticeable artifacts due to persistence of the human visual system. Figure 3 demonstrates the plot of $R_{q}(q)$ versus quantization step size $q$ for the standard Akiyo test sequence. Hence, the resulting joint rate function $R(q, t)$ is given in terms of the normalized rate functions $R_{t}(t), R_{q}(q)$ as

$$
\begin{aligned}
R(q, t) & =R_{\max } R_{t}(t) R_{q}(q) \\
& =R_{\max }\left(\frac{1-e^{-c t / t_{\max }}}{1-e^{-c}}\right) e^{d\left(1-q / q_{\min }\right)},
\end{aligned}
$$

where $R_{\max }$ is the bitrate of the highest quality video sequence corresponding to encoding at frame rate $t_{\max }$ and quantization parameter $q_{\text {min }}$. The plot in Figure 4 demonstrates that the proposed rate model closely follows the observed rate. Videos coded at lower values of quantization parameter $q \in[1,15]$ result in an exponential increase in bitrate and hence are not suitable for transmission in bandwidth constrained wireless scenarios. Further, we limit the quantization parameter to $q_{\max }=40$, as higher values lead to significant degradation of video quality.

Similarly, the normalized video quality functions $Q_{t}(t), Q_{q}(q)$ with respect to the frame rate $t$ and quantization parameter $q$, respectively can be modeled as

$$
Q_{t}(t)=\frac{1-e^{-a t / t_{\max }}}{1-e^{-a}}, \quad Q_{q}(q)=\beta q+\gamma .
$$

The quality function $Q_{t}(t)$ describes the variation in quality as a function of the frame rate $t$ and is characterized by the parameter value $a$. This value is higher in videos with lower motion content when compared to videos with higher degree of motion. The function $Q_{q}(q)$ is well approximated as a linear function of the quantization parameter $q$ as demonstrated in Figure 5. The parameters $\beta, \gamma$ are derived by fitting a linear model to video quality at the points $q=15$ and $q=35$ using the models specified in [5], while parameter values $a$ are given in [5] for CIF resolution and have been linearly extrapolated for the remaining videos of different resolutions with the values given in [10]. The resulting video quality is described by the product function:

$$
\begin{aligned}
Q(q, t) & =Q_{\max } Q_{t}(t) Q_{q}(q) \\
& =Q_{\max }\left(\frac{1-e^{-a t / t_{\max }}}{1-e^{-a}}\right)(\beta q+\gamma) .
\end{aligned}
$$

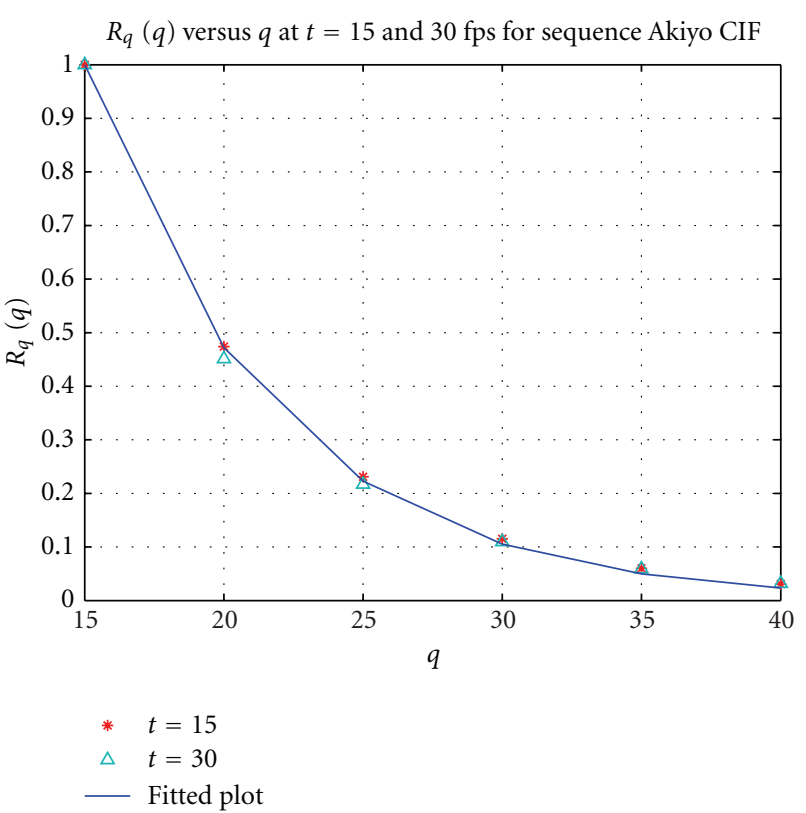

FIgURE 3: Normalized rate $R_{q}(q)$ versus $q$ at $t=15,30 \mathrm{fps}$ for sequence Akiyo (CIF).

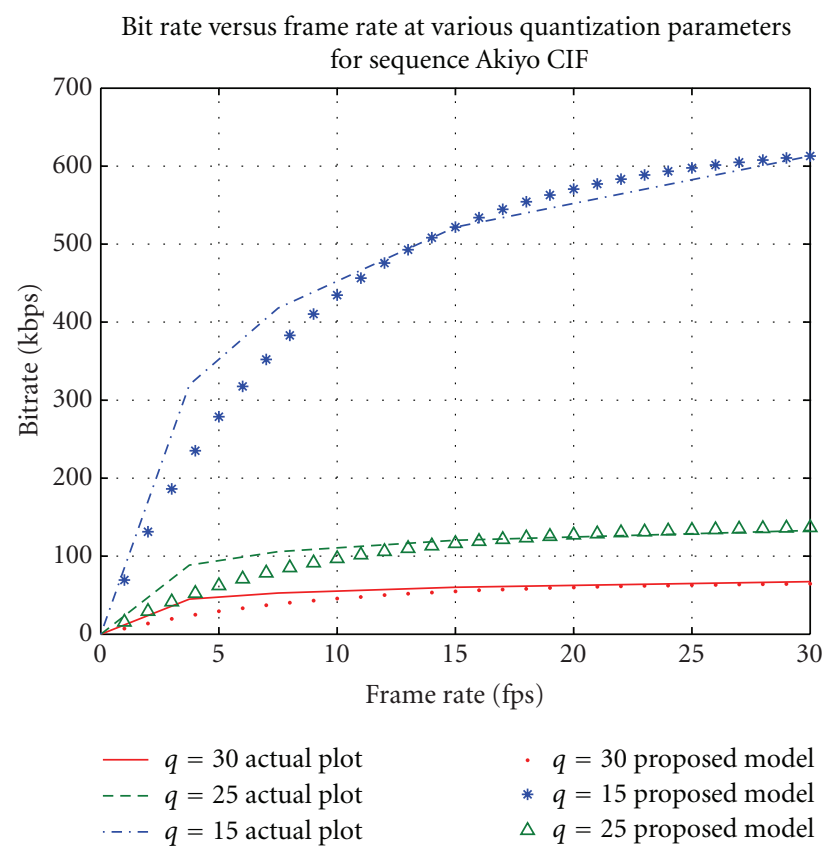

Figure 4: Plot showing proposed bitrate following actual bitrate at $q=15,25$, and 30 .

The constant $Q_{\max }$ is the quality when the video is coded at $t_{\max }, q_{\min }$ and can be normalized as $Q_{\max } \triangleq 100$. For a fixed frame rate $t_{f}$ fps, the quality depends exclusively on the quantization parameter given by $Q_{q}(q)$. This function can then be employed as a handle to maximize the video quality. 


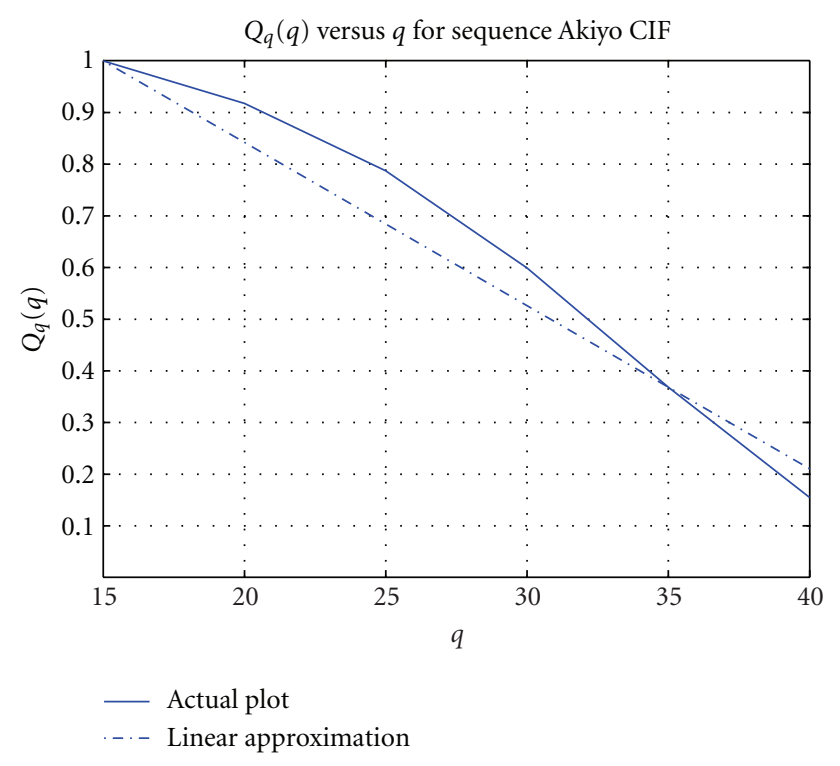

FIGURE 5: Video quality $Q_{q}(q)$ versus $q$ for the video sequence Akiyo (CIF).

\section{Optimal Bitrate Calculation}

Let $R_{S}$ denote the total symbol rate corresponding to all the subcarriers of the WiMAX OFDMA frame and $n_{i}, 1 \leq i \leq$ $N$, the number of users corresponding to the $i$ th multicast group. Let $Q^{i}\left(q_{i}, t_{f}\right), R^{i}\left(q_{i}, t_{f}\right)$ represent the quality and rate of the $i$ th video sequence corresponding to the quantization parameter $q_{i}$ for a given frame rate $t_{f}$. Let $m_{i}$ be the number of bits per symbol, that is, modulation order and $r_{i}$ the code rate of the $i$ th user in the unicast scenario. Let $\xi_{i}$ denote the bit-error introduced resulting in a required bitrate $R^{i}\left(q_{i}, t_{f}\right) /\left(1-\xi_{i}\right)$ for the $i$ th video sequence. The optimization criterion for rate allocation towards video quality maximization can be formulated as

$$
\begin{aligned}
\max . & \sum_{i=1}^{N} n_{i} Q^{i}\left(q_{i}, t_{f}\right) \\
\text { subject to } & \sum_{i=1}^{N} \frac{R^{i}\left(q_{i}, t_{f}\right)}{m_{i} r_{i}\left(1-\xi_{i}\right)} \leq R_{S} \\
& q_{\min } \leq q_{i} \leq q_{\max }, \quad 1 \leq i \leq N .
\end{aligned}
$$

The Lagrangian $L(\bar{q}, \lambda, \bar{\mu}, \bar{\delta})$ of the above optimization problem can be expressed using the Lagrange multipliers $\lambda, \mu_{i}$, $\delta_{i}, 1 \leq i \leq N$ as

$$
\begin{aligned}
L(\bar{q}, \lambda, \bar{\mu}, \bar{\delta})= & \sum_{i=1}^{N} n_{i} Q_{\max } Q_{t}^{i}\left(t_{f}\right)\left(\beta_{i} q_{i}+\gamma_{i}\right) \\
& +\lambda\left(\sum_{i=1}^{N} k_{i} e^{d_{i}\left(1-q_{i} / q_{\min }\right)}-R_{S}\right) \\
& +\sum_{i=1}^{N} \mu_{i}\left(q_{i}-q_{\max }\right)+\sum_{i=1}^{N} \delta_{i}\left(q_{\min }-q_{i}\right),
\end{aligned}
$$

\section{VCG-Based Video Resource Allocation}

In this section, we present the VCG pricing- $[12,13]$

based TF resource allocation procedure for video quality

where $k_{i} \triangleq\left(R_{\max }^{i} / m_{i} r_{i}\left(1-\xi_{i}\right)\right)\left(\left(1-e^{-c_{i} t_{f} / t_{\max }}\right) /\left(1-e^{-c_{i}}\right)\right)$, and the quantity $R_{\max }^{i}$ is the maximum bitrate corresponding to the $i$ th video. The KKT conditions for the above Lagrangian optimization criterion with $\overline{\mu_{i}} \succeq 0, \overline{\delta_{i}} \geq 0$, can be formulated as follows:

$$
\begin{gathered}
n_{i} Q_{\max } Q_{t}^{i}\left(t_{f}\right) \beta_{i}-\lambda k_{i}\left(\frac{d_{i}}{q_{\min }}\right) e^{d_{i}\left(1-q_{i} / q_{\min }\right)}+\mu_{i}-\delta_{i}=0, \\
\sum_{i=1}^{N} k_{i} e^{d_{i}\left(1-q_{i} / q_{\min }\right)} \leq R_{S}, \\
\lambda\left(\sum_{i=1}^{N} k_{i} e^{d_{i}\left(1-q_{i} / q_{\min }\right)}-R_{S}\right)=0,
\end{gathered}
$$

where the last condition above follows from the complementary slackness of the inequality constraint. Assuming $\mu_{i}=0$ and $\delta_{i}=0$, the expression for the optimal Lagrange multiplier $\lambda^{*}$ can be derived as

$$
\lambda^{*}=\frac{q_{\min }}{R_{S}}\left(\sum_{j=1}^{N} n_{j} Q_{\max } Q_{t}^{j}\left(t_{f}\right) \frac{\beta_{j}}{d_{j}}\right) .
$$

Substituting the value of $\lambda^{*}, \mu_{i}$ and $\delta_{i}$ in the first KKT equation yields the closed form expression for the optimal quantization parameter $q_{i}^{*}$ given as

$$
\begin{aligned}
q_{i}^{*} & =q_{\min }\left(1-\frac{1}{d_{i}} \ln \left(\frac{Q_{\max } Q_{t}^{i}\left(t_{f}\right) q_{\min } \beta_{i} m_{i} r_{i}\left(1-\xi_{i}\right) n_{i}}{R_{\max }^{i} R_{t}^{i}\left(t_{f}\right) \lambda^{*} d_{i}}\right)\right) \\
& =q_{\min }\left(1-\frac{1}{d_{i}} \ln \left(\frac{R_{S}}{k_{i}} \frac{n_{i} Q_{t}^{i}\left(t_{f}\right) \beta_{i}\left(d_{i}\right)^{-1}}{\sum_{j=1}^{N} n_{j} Q_{t}^{j}\left(t_{f}\right) \beta_{j}\left(d_{i}\right)^{-1}}\right)\right) .
\end{aligned}
$$

Substituting $q_{i}^{*}$ in (2) and (4) gives the required bitrate and maximum quality for each video. Figure 6 shows the optimal video quality versus bitrate plot for the video sequence Akiyo (CIF) as a function of the maximum rate $R_{S}$ at various frame rates. This corresponds to the unicast scenario in the above frame work with $N=1$. As can be seen, the video quality is near $100 \%$ for bitrates in the range of $500-600 \mathrm{Kbps}$. At lower frame rates $t$, it can be seen from (4) that the quality $Q$ at higher bitrates is lower than $100 \%$ because the normalized quality function $Q_{t}(t) \ll 1$ for $t=$ $3.75,7.5 \mathrm{fps}$.

Based on the above analysis, we present an algorithm for fast computation of the optimal quantization parameters $q_{i}^{*}$ employing the closed form expression in (9). This algorithm has a very low computational complexity and hence can be employed for rapid computation of the optimal parameters. Algorithm 1 is described for the general case of multicast video transmission. This can be readily employed for the unicast scenario by substituting $n_{i}=1$. 


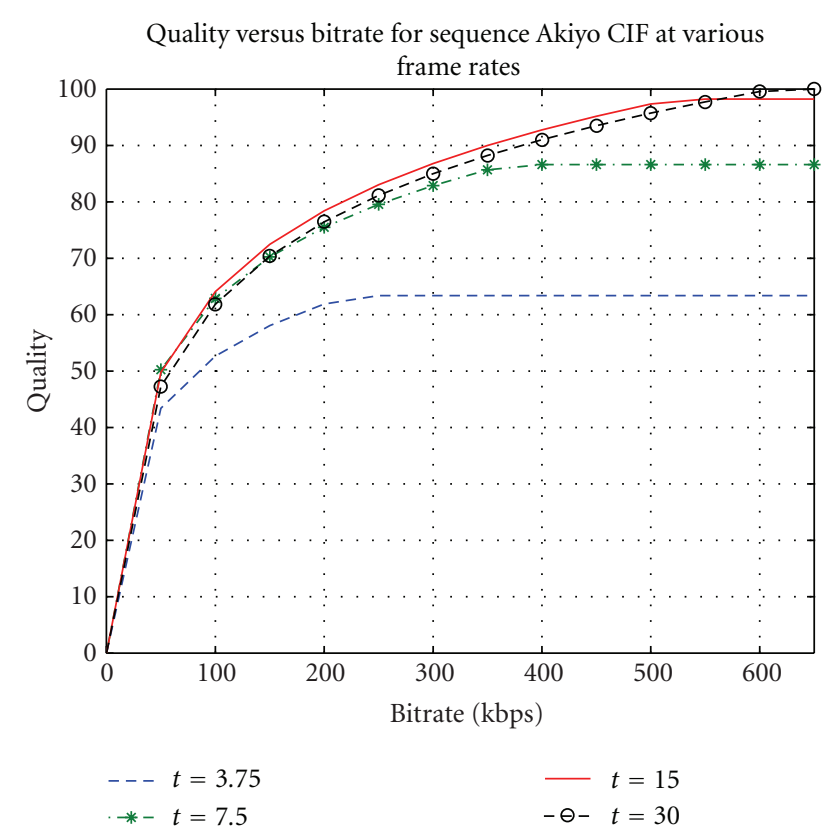

FIGURE 6: Quality versus Bitrate for sequence Akiyo CIF at various frame rates.

maximization. We consider the variation of the net VCGallocated utility as a function of the reported parameters $d$ and $\beta$ and demonstrate that its application in video rate and quality optimization leads to maximization of the net utility function. The utility function in this context of unicast/multicast video transmission, is the quality of video, which is given as a function of the quantization parameter in (4). The player/user might misreport the parameter values and subvert the allocation towards achieving disproportionate bitrate and therefore high-quality video at the cost of reduced quality to the other users. The overall utility and efficient allocation of bitrate to different videos is thus compromised. Such malicious users are penalized through the VCG auction-based TF resource pricing, which automatically leads to higher pricing and net utility reduction for the users misreporting the characteristic video parameter values. Let the actual and the reported utility functions of the $i$ th user be denoted by $Q^{i}\left(q_{i}, t_{f}\right)$ and $M^{i}\left(q_{i}, t_{f}\right)$, respectively. The QoS enforcer determines the optimal allocation as per the reported utility functions $M^{i}\left(q_{i}, t_{f}\right)$. Let $\mathbf{q}^{*}$ denote the optimal quantization parameter allocation determined from the above convex optimization frame work. Also, let the quantity $Y_{i}\left(M_{-i}()\right)$ for the $i$ th user be defined as a function of the $N-1$ utility functions $M^{j}\left(q_{j}, t_{f}\right)$ for all $j \neq i$ as

$$
Y_{i}\left(M_{-i}()\right)=\max _{\substack{q \\ \underline{j}}} \sum_{\substack{j=1 \\ j \neq i}}^{N} M^{j}\left(q_{j}, t_{f}\right) .
$$

The VCG auction price $p_{i}$ of the allocated TF resources for video transmission to the $i$ th user is given by the relation:

$$
p_{i}=Y_{i}\left(M_{-i}()\right)-L_{i}\left(q^{*}\right)
$$

where the quantity $L_{i}\left(q^{*}\right)$ is defined as $L_{i}\left(q^{*}\right) \triangleq$ $\sum_{\substack{j=1 \\ j \neq i}}^{N} M^{j}\left(q_{j}^{*}, t_{f}\right)$. It can be readily demonstrated that such a VCG auction-based pricing scheme results in serving appropriate retribution to the dishonest subscribers and service providers. Consider the net utility $Z_{i}$ of the $i$ th player given as

$$
Z_{i} \triangleq Q^{i}\left(q_{i}^{*}, t_{f}\right)-p_{i}
$$

which is essentially the raw video quality adjusted for the price paid towards serving the users. The above net utility $Z_{i}$ can be expressed in terms of the true utility function $Q^{i}\left(q_{i}, t_{f}\right)$ and the reported utility function $M^{i}\left(q_{i}, t_{f}\right)$ as

$$
Z_{i}=\underbrace{Q^{i}\left(q_{i}^{*}, t_{f}\right)+\sum_{\substack{j=1 \\
j \neq i}}^{N} M^{j}\left(q_{j}^{*}, t_{f}\right)}_{U_{i}^{*}\left(\mathbf{q}_{i}^{*}\right)}-\max _{\substack { q \\
\begin{subarray}{c}{j=1 \\
j \neq i{ q \\
\begin{subarray} { c } { j = 1 \\
j \neq i } }\end{subarray}}^{N} M^{j}\left(q_{j}, t_{f}\right) .
$$

The last term $\max _{\underline{q}} \sum_{\substack{j=1 \\ j \neq i}}^{N} M^{j}\left(q_{j}, t_{f}\right)$ in the above expression is independent of the reported utility function of the $i$ th user. Hence, it can be observed that $U_{i}\left(\mathbf{q}^{*}\right)$ for player $i$ is maximum for the allocated resource $\mathbf{q}^{*}$, calculated as per the optimization framework, only when the reported utility function $M^{i}\left(q_{i}{ }^{*}, t_{f}\right)$ coincides with the true utility function $Q^{i}\left(q_{i}^{*}, t_{f}\right)$. Thus, the VCG procedure effectively punishes malicious users who deliberately misrepresent their video parameters. This TF resource allocation based on the VCG procedure is applied to all the $N$ players/service providers participating in the given scenario. We now present Algorithm 2 for computing the VCG parameters $q_{i}^{*}$ and $p_{i}$ below.

\section{Simulation Results}

We present simulation results to illustrate the performance of the proposed optimal schemes for OFDMA video transmission employing the DL/UL PUSC (partial usage of subcarriers) diversity permutation scheme used for subcarrier channelization in WiMAX. We consider the WiMAX profile with bandwidth $B=20 \mathrm{Mhz}$, OFDMA frame time $T=$ $10 \mathrm{~ms}$ (50\% split for UL and DL traffic, i.e., $5 \mathrm{~ms}$ subframe for DL and UL) and number of subcarriers $N_{S}=2048$ [19]. The number of data subcarriers is $N_{d}=1440$ with each DL frame consisting of 44 OFDM symbols for data transmission out of the total available 48 symbols. Hence, the effective downlink symbol rate is $R_{S}=44 \times 1440 \times(10 \times$ $\left.10^{-3}\right)^{-1}=6.336 \mathrm{Msym} / \mathrm{s}$. We assume that the distorting effects of interchannel interference and doppler effect are negligible to due robust signal processing at the physical layer.

5.1. Optimal 4G Video Resource Allocation. We consider the optimal time-frequency resource allocation for video transmission in the context of the WiMAX system described above. We begin with a unicast video transmission scenario, where each of the $N(=9)$ standard video test sequences [20] of various spatial resolutions (QCIF, CIF, and 4CIF) listed 


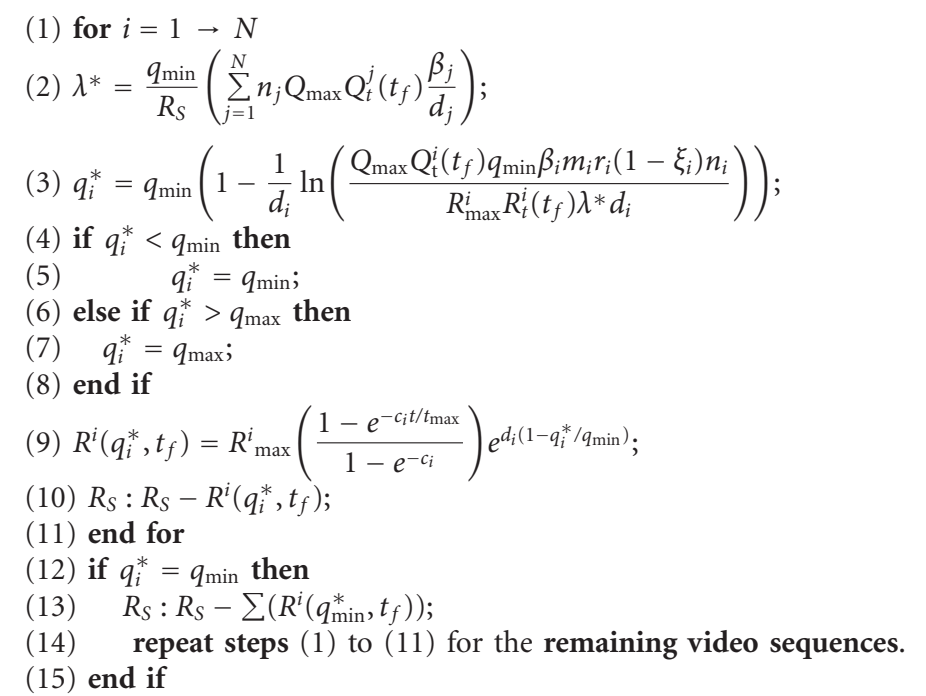

Algorithm 1: Optimal quality.

(1) compute $R^{i}\left(q_{i}^{*}, t_{f}\right)$ and $Q^{i}\left(q_{i}^{*}, t_{f}\right)$ employing Algorithm 1;

(2) set $q_{i}^{*}=\hat{q}_{i}$ using $\left\{R^{i}, d_{t}\right\}$ or $\left\{Q^{i}, \beta_{t}\right\}$ to avoid violation of constraints;

(3) compute $Y_{i}\left(M_{i}()\right)=\max _{\underline{q}} \sum_{\substack{j=1 \\ j \neq i}}^{N} M^{j}\left(q_{j}, t_{f}\right)$ employing (5);

(4) compute $L_{i}\left(q^{*}\right)$;

(5) $p_{i}=Y_{i}\left(M_{-i}()\right)-L_{i}\left(q^{*}\right)$;

(6) repeat steps (1) to (4) with different $d$ or/and $\beta$.

(7) select minimum $p_{i}$.

Algorithm 2: Algorithm for $q_{i}^{*}$ and $p_{i}$.

in Table 1 along with the associated values of the video characteristic parameters $a_{i}, c_{i}, d_{i}, \beta_{i}$, and $\gamma_{i}$ are streamed to individual users. Table 2 presents the symbol rate and quality for optimal and equal symbol rate allocation. The videos under consideration have different resolutions and varying degrees of motion. The values of the modulation index $m_{i}$ for each user are chosen randomly from the set $\{1,2,4,6\}$ corresponding to the standard WiMAX modulation formats BPSK, QPSK, 16-QAM, and 64-QAM, respectively. The coding rates $r_{i}$ are similarly chosen randomly from the set $\{1 / 2,2 / 3,3 / 4,5 / 6\}$ of standard WiMAX coding rates. The optimal video quality maximizing bitrate allocation and the associated quantization parameters $q_{i}^{*}$ are computed by solving the optimization problem in (5) employing the standard CVX based convex solver [21] and the closed form solution based scheme in Algorithm 1. The corresponding per video sequence normalized quality is listed in Table 1 for both the optimal and equal symbol rate allocation schemes at $t=30 \mathrm{fps}$ from which it can be readily seen that the optimal resource allocation scheme outperforms the suboptimal equal resource allocation scheme. Figure 7 shows the comparison of these schemes for the above unicast scenario at various values of symbol rate $R_{S}$, clearly demonstrating the efficiency of the optimal allocation scheme described in Section 3. Further, the optimal resource allocation computed employing the closed form solution in (9) and the associated fast algorithm described in Algorithm 1 achieve a performance close to that of the CVX solver, thereby verifying the theoretical analysis.

Figure 8 shows the comparison of these schemes for multicast scenarios with the number of multicast subscribers chosen randomly from the set $30 \leq n_{i} \leq 100$ at frame rate $t=30 \mathrm{fps}$. The bit-errors $\xi_{i}$ are assumed to be random in the interval $\left[10^{-3}, 10^{-5}\right]$. The parameters $m_{i}$ and $r_{i}$ for each multicast group are chosen randomly as described in the unicast scenario. Similar to the unicast scenario, it can be observed that optimal resource allocation results in progressively larger gains compared to the suboptimal equal resource allocation. Further, the net normalized video qualities for both the resource allocation schemes in the standard WiMAX multicast scenario described above with rate $R_{S}=6.336 \mathrm{Msym} / \mathrm{s}$ are given in Table 3 for each of the frame rates $t=15$ and $t=30 \mathrm{fps}$. It can be clearly seen that the optimal allocation results in a significant enhancement of approximately $6.5 \%$ in the video quality over equal resource allocation. We schematically represent the optimal and equal allocation of time/frequency resources of the OFDMA symbol for unicast transmission in 
TABle 1: Parameter values and $R_{\max }$.

\begin{tabular}{|c|c|c|c|c|c|c|}
\hline Sequence & $a_{i}$ & $c_{i}$ & $d_{i}$ & $\beta_{i}$ & $\gamma_{i}$ & $R_{\max }^{i}$ \\
\hline Foreman CIF & 7.7000 & 2.0570 & 2.2070 & -0.0298 & 1.4475 & 3046.3 \\
\hline Akiyo CIF & 8.0300 & 3.4910 & 2.2520 & -0.0316 & 1.4737 & 612.85 \\
\hline Football CIF & 5.3800 & 1.3950 & 1.4900 & -0.0258 & 1.3872 & 5248.9 \\
\hline Crew CIF & 7.3400 & 1.6270 & 1.8540 & -0.0393 & 1.5898 & 4358.2 \\
\hline City CIF & 7.3500 & 2.0440 & 2.3260 & -0.0346 & 1.5196 & 2775.5 \\
\hline Akiyo QCIF & 5.5600 & 4.0190 & 1.8320 & -0.0316 & 1.4737 & 139.63 \\
\hline Foreman QCIF & 7.1000 & 2.5900 & 1.7850 & -0.0298 & 1.4475 & 641.73 \\
\hline City 4CIF & 8.4000 & 1.0960 & 2.3670 & -0.0346 & 1.5196 & 20900 \\
\hline Crew 4CIF & 7.3400 & 1.1530 & 2.4050 & -0.0393 & 1.5898 & 18021 \\
\hline
\end{tabular}

TABLE 2: Allocation of symbols in an OFDM frame for unicast at $t=30 \mathrm{fps}$.

\begin{tabular}{|c|c|c|c|c|c|c|}
\hline Sequence & $m_{i}$ & $r_{i}$ & $\begin{array}{l}\text { Equal symbol rate } \\
\text { selction (ksps) }\end{array}$ & $Q_{i} / Q_{\max }$ & $\begin{array}{l}\text { Optimal symbol rate } \\
\text { allocation (ksps) }\end{array}$ & $Q_{i} / Q_{\max }$ \\
\hline Foreman CIF & 1 & $5 / 6$ & 704 & 0.666 & 685 & 0.660 \\
\hline Akiyo CIF & 2 & $2 / 3$ & 704 & 1.00 & 460 & 1.00 \\
\hline Football CIF & 1 & $2 / 3$ & 704 & 0.372 & 877 & 0.430 \\
\hline Crew CIF & 1 & $5 / 6$ & 704 & 0.362 & 1074 & 0.496 \\
\hline City CIF & 1 & $2 / 3$ & 704 & 0.602 & 754 & 0.618 \\
\hline Akiyo QCIF & 4 & $1 / 2$ & 704 & 1.00 & 70 & 1.00 \\
\hline Foreman QCIF & 1 & $3 / 4$ & 704 & 0.951 & 847 & 0.997 \\
\hline City 4CIF & 4 & $2 / 3$ & 704 & 0.471 & 741 & 0.482 \\
\hline Crew 4CIF & 1 & $1 / 2$ & 704 & 0.034 & 828 & 0.074 \\
\hline
\end{tabular}

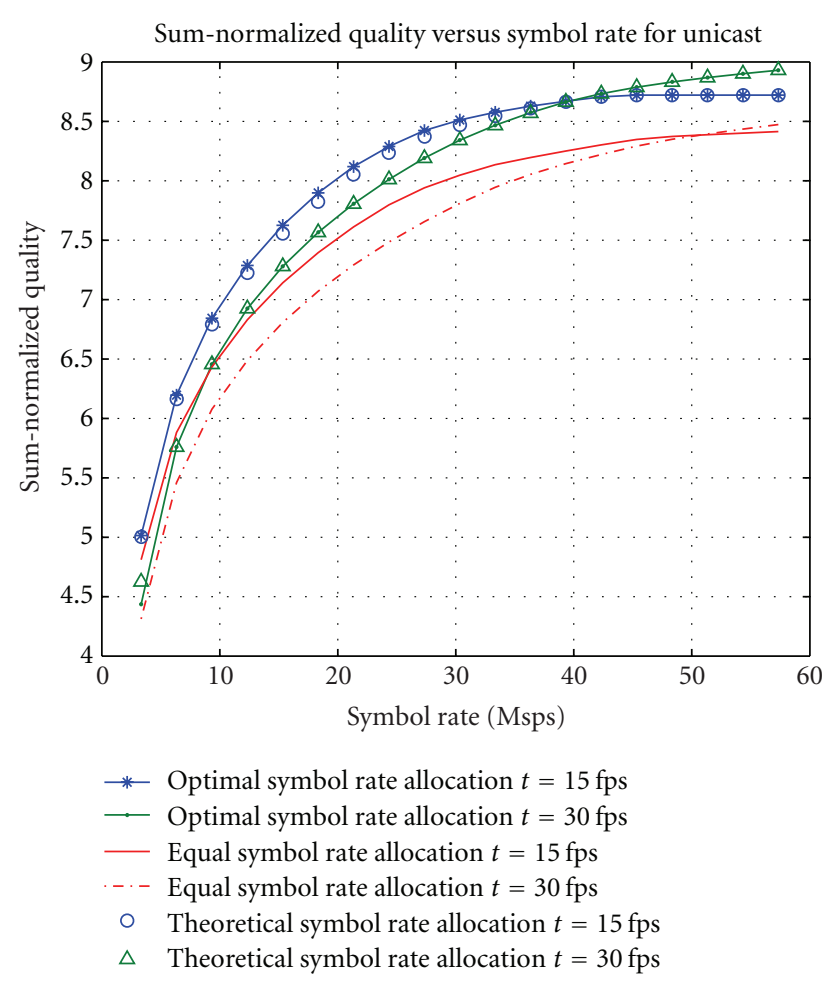

FIGURE 7: Unicast: sum-normalized quality versus symbol rate at $t=15$ and $30 \mathrm{fps}$.

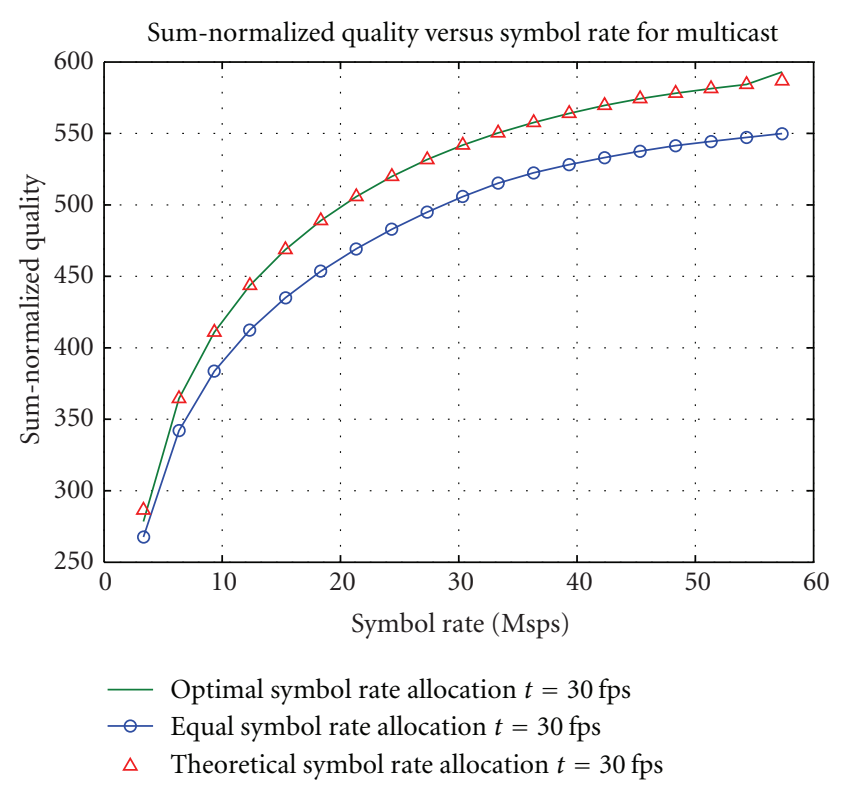

FIGURE 8: Multicast: sum normalized quality versus symbol rate at $t=15$ and $30 \mathrm{fps}$.

Figures 9 and 10, respectively with each shade representing the portion of the DL subframe allocated to a particular video sequence belonging to the set under 


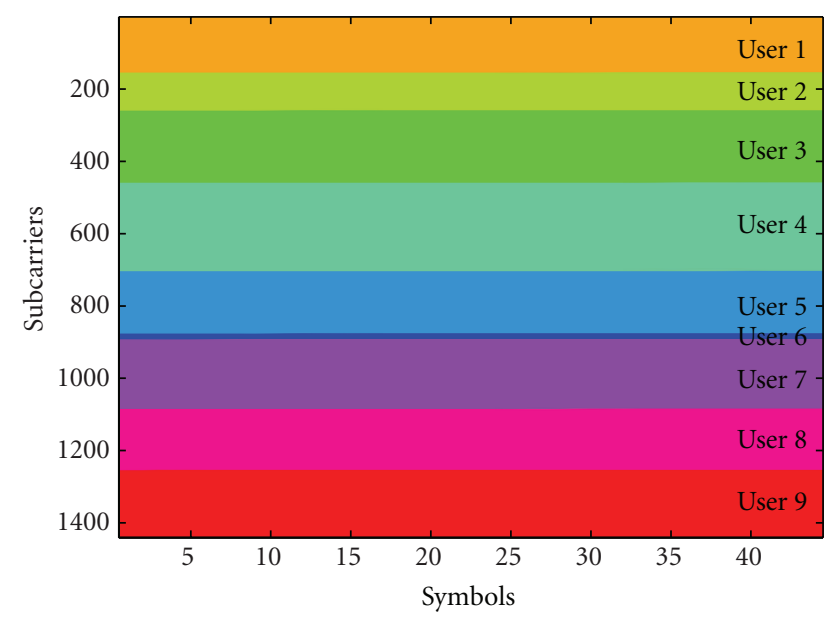

FIGURE 9: Allocation of symbols to videos with optimal allocation.

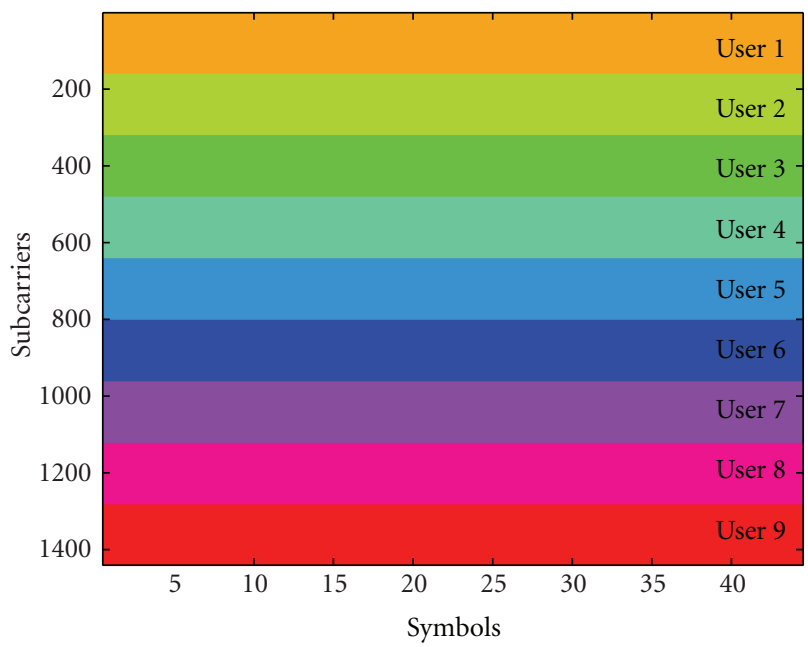

FIGURE 10: Allocation of symbols to videos with equal symbol rate allocation.

TABLE 3: Comparison of quality at frame rates for multicast $t=15$ and $t=30 \mathrm{fps}$.

\begin{tabular}{lcc}
\hline Method & $\begin{array}{c}\text { Sum } Q / Q_{\max } \text { at } \\
15 \mathrm{fps}\end{array}$ & $\begin{array}{c}\text { Sum } Q / Q_{\max } \text { at } \\
30 \mathrm{fps}\end{array}$ \\
\hline Optimal symbol rate & 395.5 & 364.9 \\
Equal symbol selection & 371.6 & 342.4 \\
\hline
\end{tabular}

consideration. Finally, we present the comparison of these schemes for unicast video transmission with $m_{i}=2$, for all $i$ at various symbol rates $R_{S}$ and varying $r_{i}$ in Figure 11. Similarly, Figure 12 shows the comparison of these schemes for unicast with $r_{i}=1 / 2$, for all $i$ at various $R_{S}$ and varying modulation order $m_{i}$. We conclude that higher modulation and coding rate provide higher net quality to the users. Overall, the optimal resource allocation algorithm proposed for OFDMA-based time-frequency resource allocation results in a significant improvement in the net video quality.

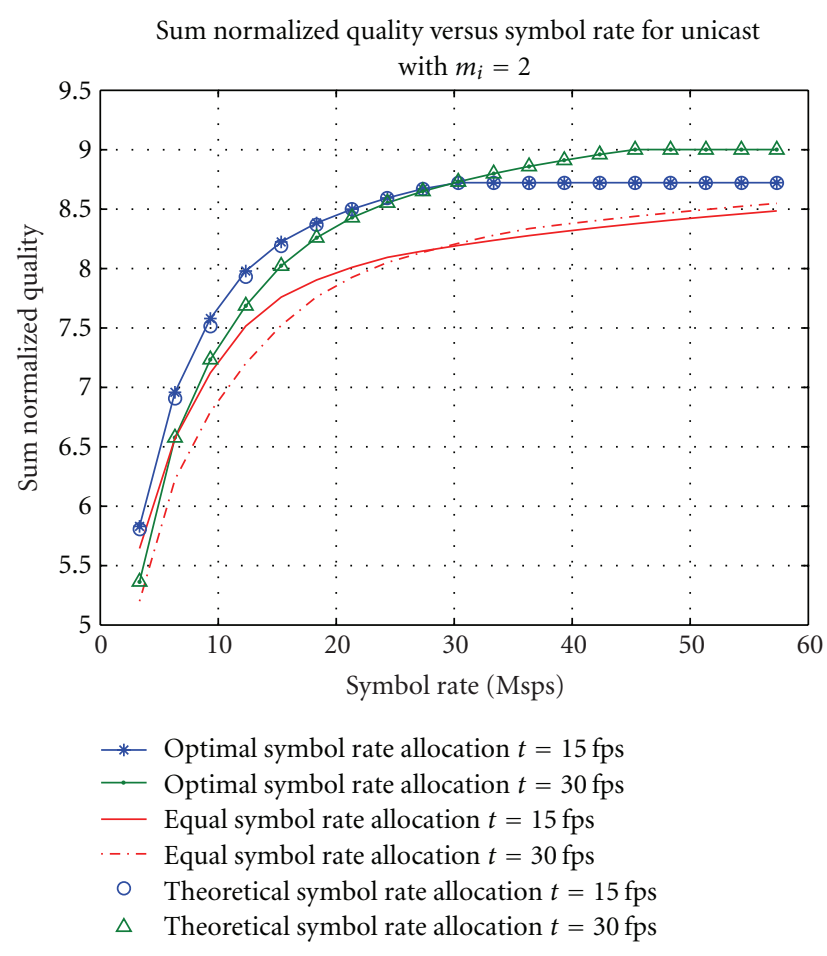

FIGURE 11: Unicast: sum normalized quality versus symbol rate at $t=15$ and 30 fps with $m_{i}=2$, for all $i$, and varying $r_{i}$.

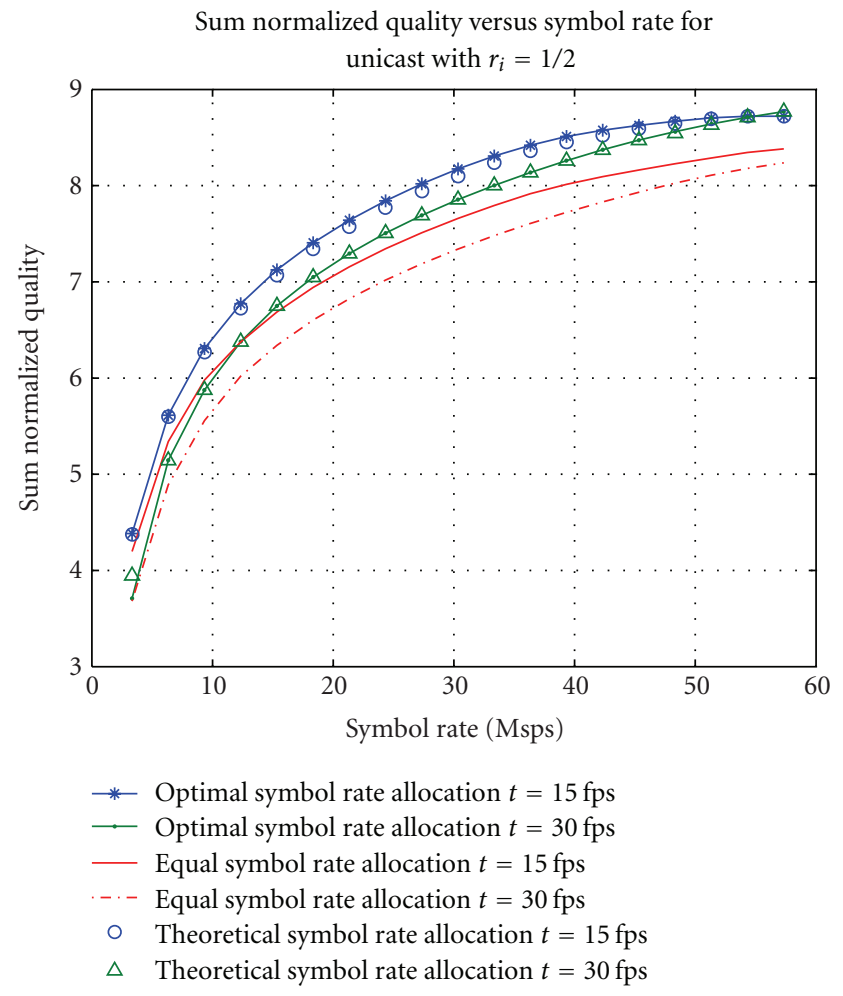

FIGURE 12: Unicast: sum normalized quality versus symbol rate at $t=15$ and $30 \mathrm{fps}$ with $r_{i}=1 / 2$, for all $i$, and varying $m_{i}$. 


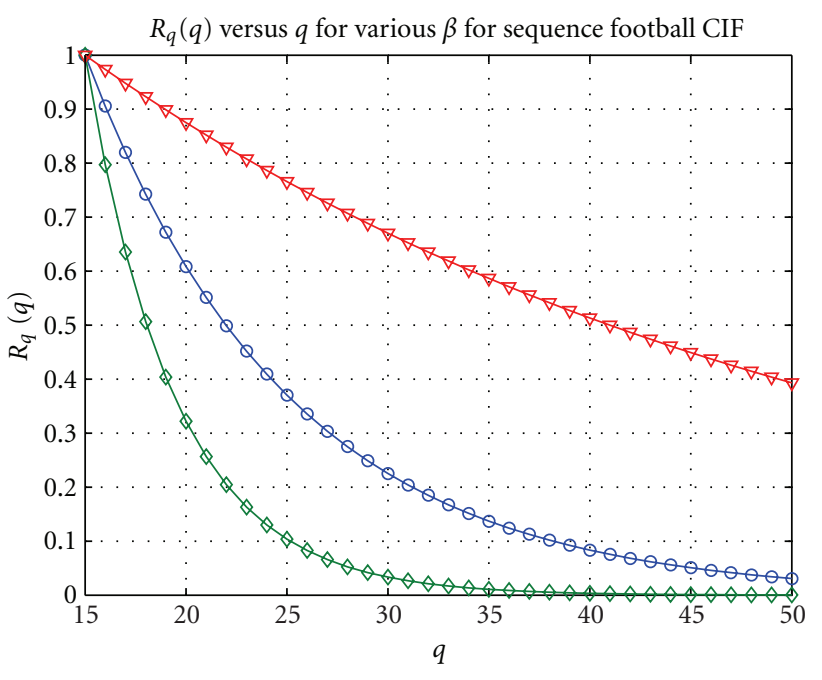

$$
\begin{array}{ll}
\multimap & d_{t}=1.49 \\
\multimap & d_{m}=3.4 \\
\rightarrow & d_{m}=0.4
\end{array}
$$

FIgURE 13: Rate versus quantization parameter at various $d$ for sequence football CIF: $d_{t}=$ true value, $d_{m}=$ misreprted value.

TABle 4: Quantization parameter and bitrate for sequence football, Case I: $d=d_{t}$, Case II: $d<d_{t}$, and Case III: $d>d_{t}$.

\begin{tabular}{lccc}
\hline Case & I & II & III \\
\hline$d_{3}$ & 1.49 & 0.4 & 3.4 \\
$q_{3}$ & 27.82 & 24.48 & 23.36 \\
$R^{3}$ & 1621.9 & 4076.6 & 789 \\
\hline
\end{tabular}

5.2. VCG Auction-Based 4G Video Resource Allocation. In this section, we study the impact of the parameters $d$ and $\beta$ on the bitrate and quality of the video. We then demonstrate the application of the proposed VCG procedure when the user misreports the parameter values. We consider $n_{i}=1, r_{\mathrm{i}}=5 / 6$, and $m_{i}=2$ for all $i$ to study the effect of misreporting $d$ and $\beta$. We consider the optimal allocation of TF resources in this scenario to the different groups and the net utility corresponding to accurate and misreporting of $d$, and $\beta$ parameters. We begin by specifically considering two separate cases in which a single subscriber of the standard test video sequence football CIF [20] misreports the parameter values $d$ (rate parameter) and $\beta$ (quality parameter). The scenario with multiple users misreporting multiple parameters is considered in the later simulations.

5.2.1. Behavior Corresponding to Misreporting $d$. In this section we illustrate the effect of false reporting of parameter $d$ for the standard football video sequence on the overall bitrate allocation. Figure 13 depicts the bitrate of the sequence Football corresponding to $i=3$, as a function of the quantization parameter $q$ for different values of the reported rate parameter $d$, where the true parameter $d_{t}=1.49$. The curves corresponding to misreporting the $d$ parameter, that is, $d_{m}=3.4>d_{t}$ and $d_{m}=0.4<d_{t}$ can be seen

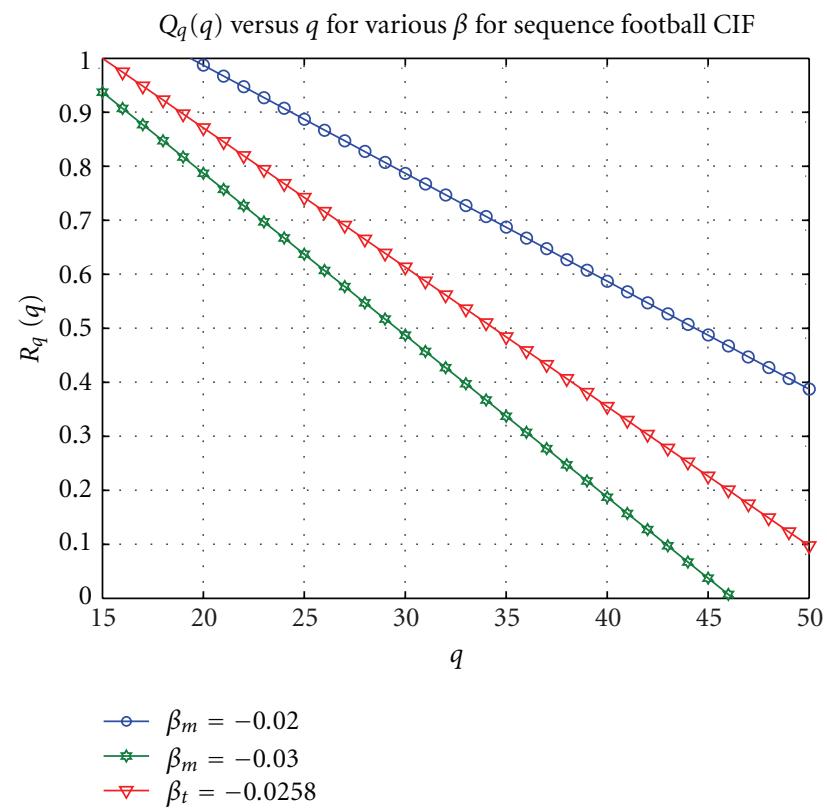

FIGURE 14: Quality versus quantization parameter at various $\beta$ for sequence football CIF: $\beta_{t}=$ true value, $\beta_{m}=$ misreprted value.

therein. Cases I, II and III in Table 4 demonstrate the allotted quantization parameter and corresponding bitrate when $d_{m}=d_{t}, d_{m}<d_{t}$ and $d_{m}>d_{t}$, respectively at $R_{S}=$ 6.336 Msps for the standard football CIF sequence. Consider the adverse scenarios, where the user/service provider reports $d_{m}=0.4<d_{t}$ shown in case II. This results in suboptimal allocation of TF resources, with a disproportionate alloation of $R^{3}\left(q_{3}, t_{f}\right)=4076.6 \mathrm{Kbps}$. This is at the cost of decrease in video quality of the rest of the users. In the later simulations, it is shown that the application of the VCG procedure ensures that such malicious users are punished through a reduction in the net utility resulting from the VCG allocation. When $d_{m}=3.4>d_{t}$ as considered in case III, the allotted bitrate $R^{3}\left(q_{3}, t_{f}\right)=789 \mathrm{Kbps}$ is much less than the rate $1621.9 \mathrm{Kbps}$ (corresponding to case I). Hence, there is no incentive for the malicious user to misreport a lower value of the parameter $d$. However, the actual video encoded with this lower value of the allocated quantization parameter $q=23.36$ will have bitrate $R^{3}\left(q_{3}, t_{f}\right)>1621.9 \mathrm{Kbps}$ (corresponding to case I) and thus results in violating the overall bitrate constraint. Hence, the malicious user in this scenario is forced to compute the quantization parameter $\hat{q}_{3}$ corresponding to the allocated bitrate of $789 \mathrm{Kbps}$ to ensure that the rate constraints are not violated. This results in lower quality $Q^{3}\left(\hat{q}_{3}, t_{f}\right)$.

5.2.2. Behavior Corresponding to Misreporting $\beta$. We now consider the effect of misreporting of the parameter $\beta$ of a video sequence on the overall TF resource allocation. Figure 14 depicts the video quality as a function of the quantization parameter $q$ for the true value $\beta_{t}=-0.0258$ and misreported values $\beta_{m}=-0.03,-0.02$. Cases I, II, and III in Table 5 show the computed quantization parameters 
TABLE 5: Quantization parameter and bitrate for sequence football Case I: $\beta=\beta_{t}$, Case II: $\beta_{m}<\beta_{t}$, and Case III: $\beta_{m}>\beta_{t}$.

\begin{tabular}{lccc}
\hline Case & I & II & III \\
\hline$\beta_{3}$ & -0.0258 & -0.03 & -0.02 \\
$q_{3}$ & 27.82 & 25.6 & 28.98 \\
$R^{3}$ & 1621.9 & 1832.2 & 1308.5 \\
\hline
\end{tabular}

Net utility at different rates for various values of $d$ for sequence football CIF

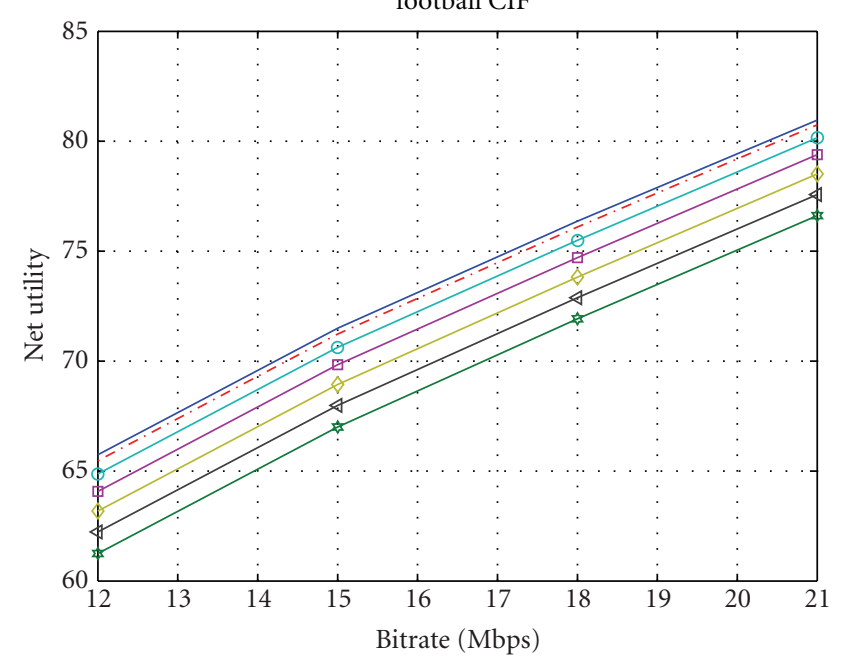

$$
\begin{aligned}
& -d_{t}=1.49 \\
& \text { - - } d_{m}=1.75 \\
& -d_{m}=2 \\
& \square d_{m}=2.25 \\
& \smile d_{m}=2.5 \\
& \triangleleft \quad d_{m}=2.75 \\
& \text { * } d_{m}=3
\end{aligned}
$$

FIGURE 15: Net utility function versus rate at various values of parameter $d$ for sequence football. CIF: $d_{t}=$ true value, and $d_{m}=$ misreprted value.

and allotted bitrates of video sequences when $\beta=\beta_{t}, \beta_{m}=$ $-0.03<\beta_{t}$ and $\beta_{m}=-0.02>\beta_{t}$, respectively at $R_{S}=6.336$ Msps for the standard video sequence football CIF. When the misreported $\beta_{m}=-0.030<-0.0258$ as in case II, the optimal bitrate allocation results in $R^{3}\left(q_{3}, t_{f}\right)=1832.2>$ $1621.9 \mathrm{Kbps}$, and the difference $1832.2-1621.9=210.3 \mathrm{Kbps}$ is obtained by taking the share of bits from other videos. Hence, similar to reporting a lower value of $d$ as seen above, the malicious user has an incentive to report a lower value of the parameter $\beta$. For case III, corresponding to $\beta>-0.0258$, the bitrate obtained $R^{3}\left(q_{3}, t_{f}\right)=1308.5<1621.9 \mathrm{Kbps}$, as shown in Table 5 . The quality $Q^{3}\left(q_{3}, t_{f}\right)$ is lower compared to the case when $\beta_{t}$ is reported. Hence, there is no incentive for the malicious user to report higher values of the quality parameter $\beta$.

5.2.3. VCG Procedure Based TF Resource Allocation. In this section, we illustrate the efficacy of the VCG procedure based resource allocation described in Section 4 towards punishing such malicious users and reducing their net utility, thereby discouraging false reporting of the video parameters. Similar to the scenarios presented above, we consider the video

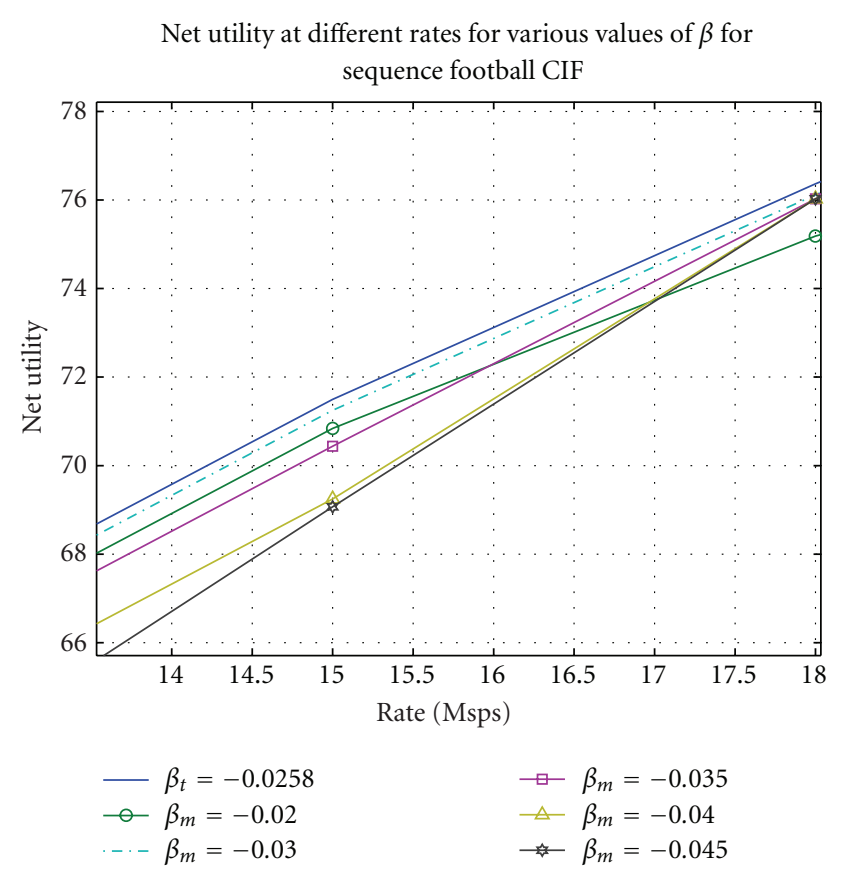

Figure 16: Net utility function versus rate at various values of parameter $\beta$ for sequence football. CIF: $\beta_{t}=$ true value, and $\beta_{m}=$ misreprted value.

streaming of $N=9$ video sequences with $m_{i} \in\{1,2,4,6\}$ and $r_{i} \in\{1 / 2,2 / 3,3 / 4,5 / 6\}$. The TF resources are allocated as per the optimal solution corresponding to the reported utility function maximization in (5) at the VCG price $p_{i}$ computed in (11). Figures 15 and 16 show the net utility function as a function of the symbol-rate $R$ corresponding to the VCG procedure based TF resource allocation for the video sequence football. It can be seen therein that the net utility function is maximum when the true parameters $d=$ $d_{t}=1.49$ and $\beta=\beta_{t}=-0.0258$. Hence, the VCG procedure penalizes the users misreporting the video characteristic parameters by decreasing their net utility. In these scenarios we only consider false reporting of a single parameter (either $d$ or $\beta$, but not both) by a single user. Below, we consider the scenario where multiple users simultaneously misreport one or more characteristic video parameters.

We assume the following misreported parameter values $\beta_{1}=-0.025, \beta_{3}=-0.020, \beta_{5}=-0.030, d_{3}=2.2, d_{4}=1.8$, $d_{6}=2.4$, with user 3 misreporting both $d$ and $\beta$ considered for simulations in Figures 17 and 18. In Figure 17 we plot the net utility of user 3 corresponding to misreporting $d_{m}=$ $2.2>d_{t}=1.49$ and several possible misreports of $\beta \neq \beta_{t}$ and $d \neq d_{t}$. It can be seen that, amongst all the net utility curves, the one corresponding to $\beta=\beta_{t}=-0.0258$ results in the maximization of net utility. Similarly, in Figure 18 we plot the net utility for the false reporting of $\beta_{m}=-0.020>$ $\beta_{t}$ and several possible misreports of the rate parameter $d$ and quality parameter $\beta$. Once again, it can be seen that reporting the true value of $d=d_{t}=1.49$ results in net utility maximization for user 3. Thus, application of the VCG procedure results in penalizing the parameter misreporting 


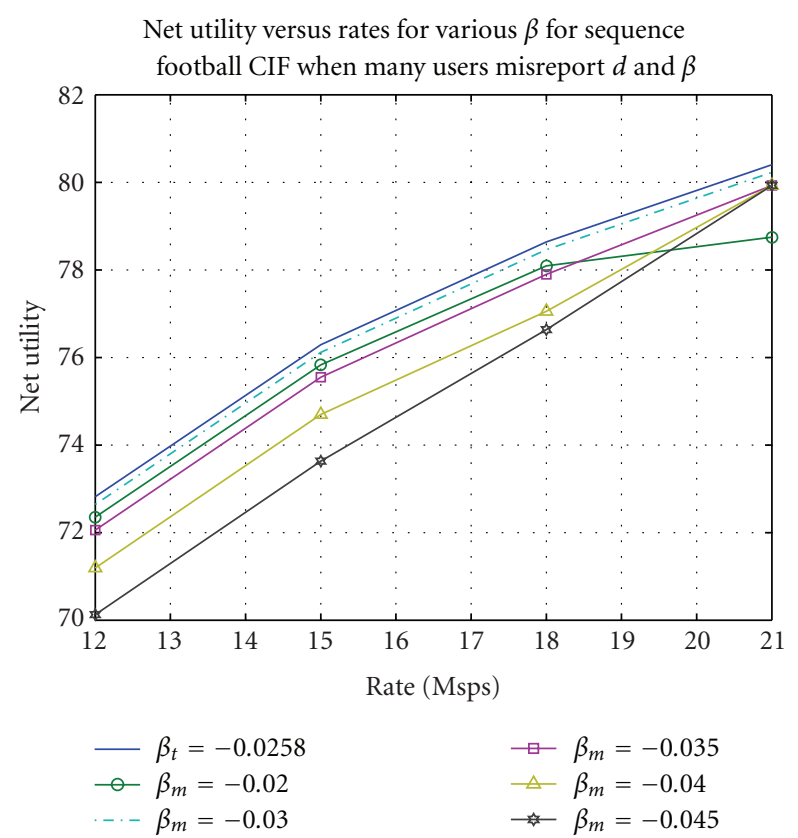

FIgURE 17: Net utility function versus rate at various values of parameter $\beta$ for sequence football CIF and other misreports: $\beta_{t}=$ true value; $\beta_{m}=$ misreprted value.

malicious users, thereby encouraging users to report the true characteristic video parameters, thus resulting in optimal TF resource allocation.

\section{Conclusion}

We presented a novel scheme for time-frequency resource allocation in OFDMA-based 4G wireless systems aimed at video quality maximization. H.264-based scalable video models have been employed to characterize the video bitrate and quality as a function of the quantization parameter $q$. Based on these models, a constrained convex optimization framework has been presented for optimal OFDMA-based unicast/multicast resource allocation. A fast algorithm based on the closed form solution of the resource optimization problem has been presented to compute the optimal quantization parameters $q_{i}^{*}$. It has been observed in simulations that the proposed optimal scheme yields a considerable improvement in the video quality. Further, the performance gains increase progressively in multicast scenarios with increasing number of subscribers. For the specific case of PUSC WiMAX with $N_{S}=2048$ subcarriers and frame time $T=10 \mathrm{~ms}$, the proposed optimal scheme obtains a quality gain of about $6.5 \%$ over the suboptimal equal symbol rate allocation scheme.

We also presented a novel VCG procedure-based approach for optimal TF resource allocation towards scalable video transmission. In conventional $4 \mathrm{G}$ resource allocation based on sum quality maximization, there is an incentive for malicious users to misreport the video quality parameters towards disproportionately high-resource allocation, thus leading to suboptimality and subversion of the scheduler

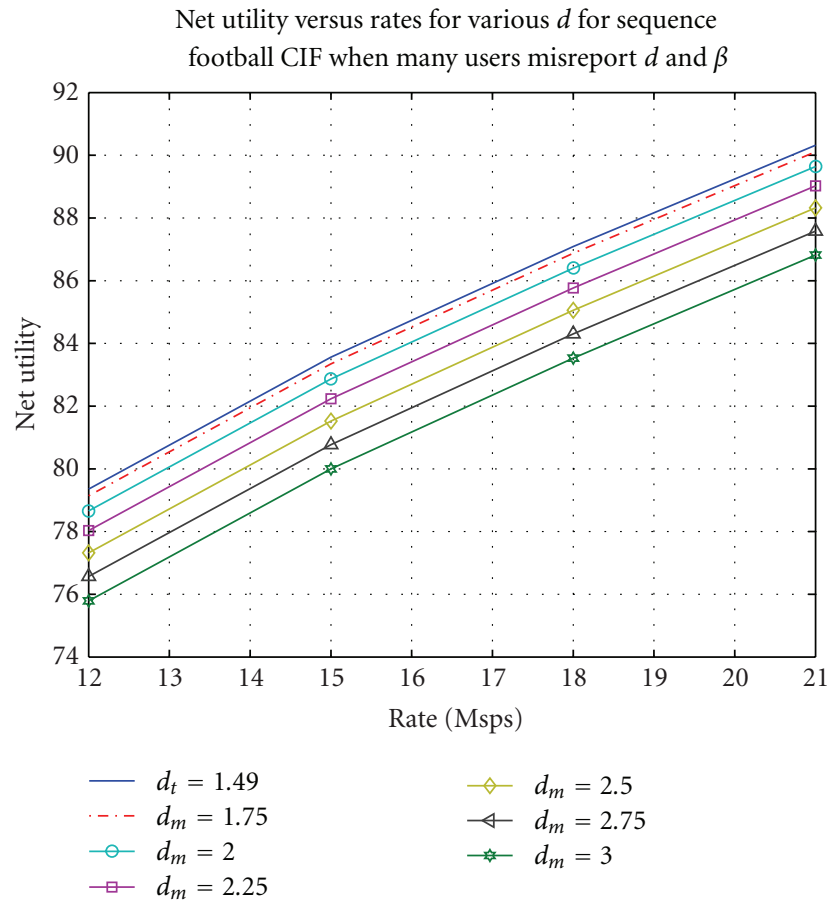

Figure 18: Net utility function versus rate at various values of parameter $d$ for sequence football CIF and other misreports: $d_{t}=$ true value; $d_{m}=$ misreprted value.

operation at the base station. The proposed VCG procedure is effective for resource allocation in such scenarios, since it punishes malicious users through pricing-based optimal resource allocation, thereby discouraging false reports. Further, the incidental outcomes of the above VCG-based allocation are the price points for the allocated TF resources. Hence, the proposed scheme can also be used as an effective TF resource pricing algorithm for use in the OSS module of the core network, which in turn leads to overall optimal resource allocation.

\section{References}

[1] D. Tse and P. Viswanath, Fundamentals of Wireless Communication, Cambridge University Press, New York, NY, USA, 2005.

[2] A. Goldsmith, Wireless Communications, Cambridge University Press, New York, NY, USA, 2005.

[3] H. Schwarz, D. Marpe, and T. Wiegand, "Overview of the scalable video coding extension of the H.264/AVC standard," IEEE Transactions on Circuits and Systems for Video Technology, vol. 17, no. 9, pp. 1103-1120, 2007.

[4] Y. Wang, Y.-Q. Zhang, and J. Ostermann, Video Processing and Communications, Prentice Hall PTR, Upper Saddle River, NJ, USA, 1st edition, 2001.

[5] Y. Wang, Z. Ma, and Y. F. Ou, "Modeling rate and perceptual quality of scalable video as functions of quantization and frame rate and its application in scalable video adaptation," in Proceedings of the 17th International Packet Video Workshop (PV'09), pp. 1-9, Seattle, Wa, USA, May 2009.

[6] Y. N. Lin, C. W. Wu, Y. D. Lin, and Y. C. Lai, "A latency and modulation aware bandwidth allocation algorithm for 
WiMAX base stations," in Proceedings of the IEEE Wireless Communications and Networking Conference (WCNC'08), pp. 1408-1413, April 2008.

[7] S. Deb, S. Jaiswal, and K. Nagaraj, "Real-time video multicast in WiMAX networks," in Proceedings of the IEEE 27th Conference on Computer Communications (INFOCOM '08), pp. 1579-1587, April 2008.

[8] J. Kim, J. Cho, and H. Shin, "Resource allocation for scalable video broadcast in wireless cellular networks," in Proceedings of the IEEE International Conference on Wireless and Mobile Computing, Networking and Communications (WiMob '05), vol. 2, pp. 174-180, August 2005.

[9] JSVM, "Joint Scalable Video Model software, Joint Video Team, version 9.19.7".

[10] Y. F. Ou, T. Liu, Z. Zhao, Z. Ma, and Y. Wang, "Modeling the impact of frame rate on perceptual quality of video," in Proceedings of the 15th IEEE International Conference on Image Processing (ICIP '08), pp. 689-692, October 2008.

[11] S. Boyd and L. Vandenberghe, Convex Optimization, Cambridge University Press, New York, NY, USA, 2004.

[12] N. Nisan, T. Roughgarden, E. Tardos, and V. Vazirani, Algorithmic Game Theory, Cambridge University Press, New York, NY, USA, 2007.

[13] "Lecture on VCG procedures," http://www.cse.iitd.ernet.in/ rahul/cs905/lecture10/index.html.

[14] V. Srivastava, J. Neel, A. Mackenzie et al., "Using game theory to analyze wireless ad hoc networks," IEEE Communications Surveys Tutorials, vol. 7, no. 4, pp. 46-56, 2005.

[15] C. Sacchi, F. Granelli, and C. Schlegel, "A qoe-oriented strategy for ofdma radio resource allocation based on minmos maximization," IEEE Communications Letters, vol. 15, no. 5, pp. 494-496, 2011.

[16] W. S. Lin, H. V. Zhao, and K. J. R. Liu, "Attack-resistant cooperation strategies in P2P live streaming social networks," in Proceedings of the 42nd Asilomar Conference on Signals, Systems and Computers (ASILOMAR '08), pp. 1373-1377, October 2008.

[17] J. Hershberger and S. Suri, "Vickrey prices and shortest paths: what is an edge worth?" in Proceedings of the 42nd Annual Symposium on Foundations of Computer Science, pp. 252-259, October 2001.

[18] D. C. Parkes and J. Shneidman, "Distributed implementations of Vickrey-Clarke-Groves mechanisms," in Proceedings of the 3rd International Joint Conference on Autonomous Agents and Multiagent Systems (AAMAS '04), pp. 261-268, July 2004.

[19] J. G. Andrews, A. Ghosh, and R. Muhamed, Fundamentals of WiMAX: Understanding Broadband Wireless Networking, Prentice Hall PTR, Upper Saddle River, NJ, USA, 2007.

[20] "Standard test video sequences," http://media.xiph.org/video/ derf/.

[21] M. Grant and S. Boyd, "CVX: matlab software for disciplined convex programming, version 1.21,” 2011, http://cvxr.com/ $\mathrm{cvx} /$. 

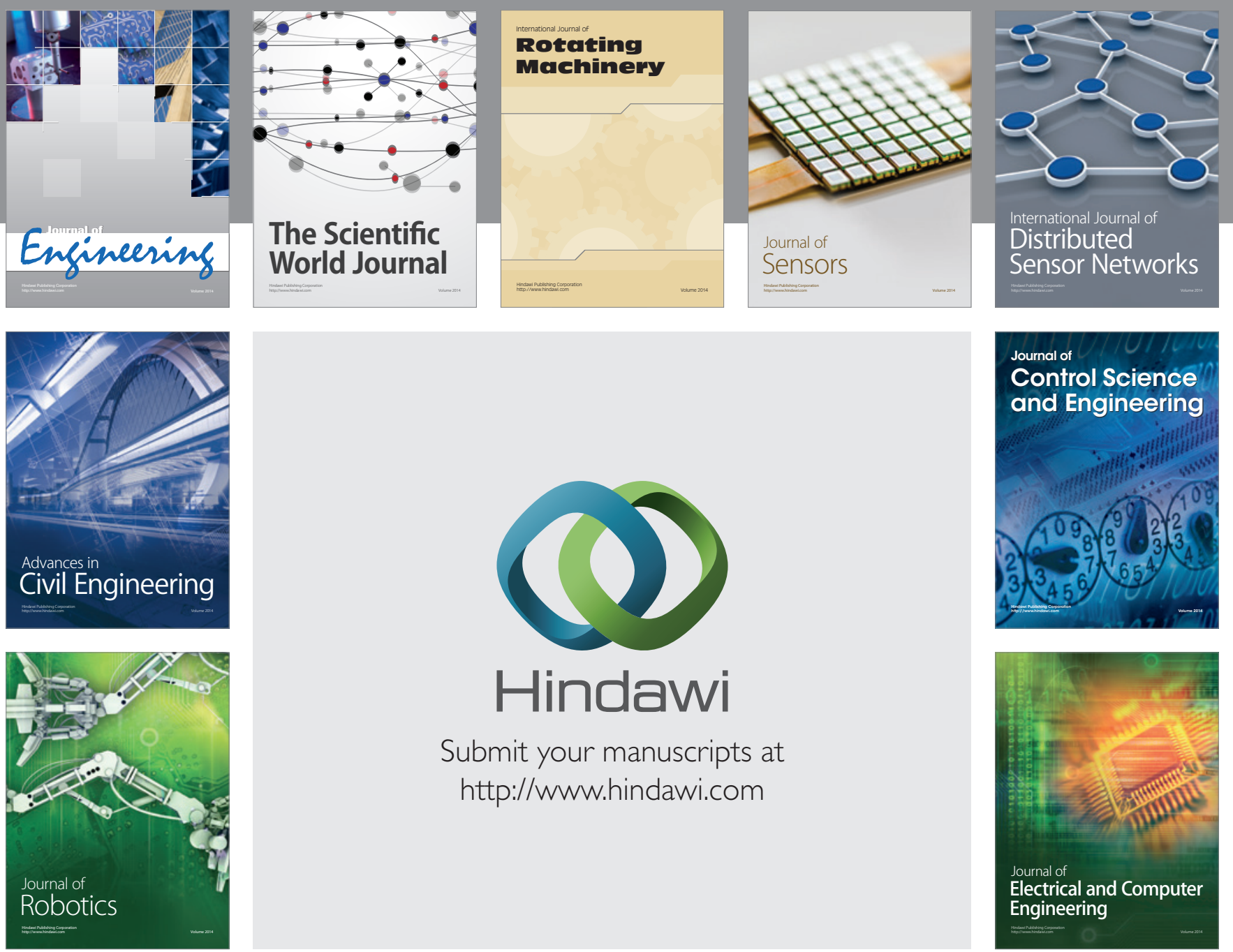

Submit your manuscripts at

http://www.hindawi.com
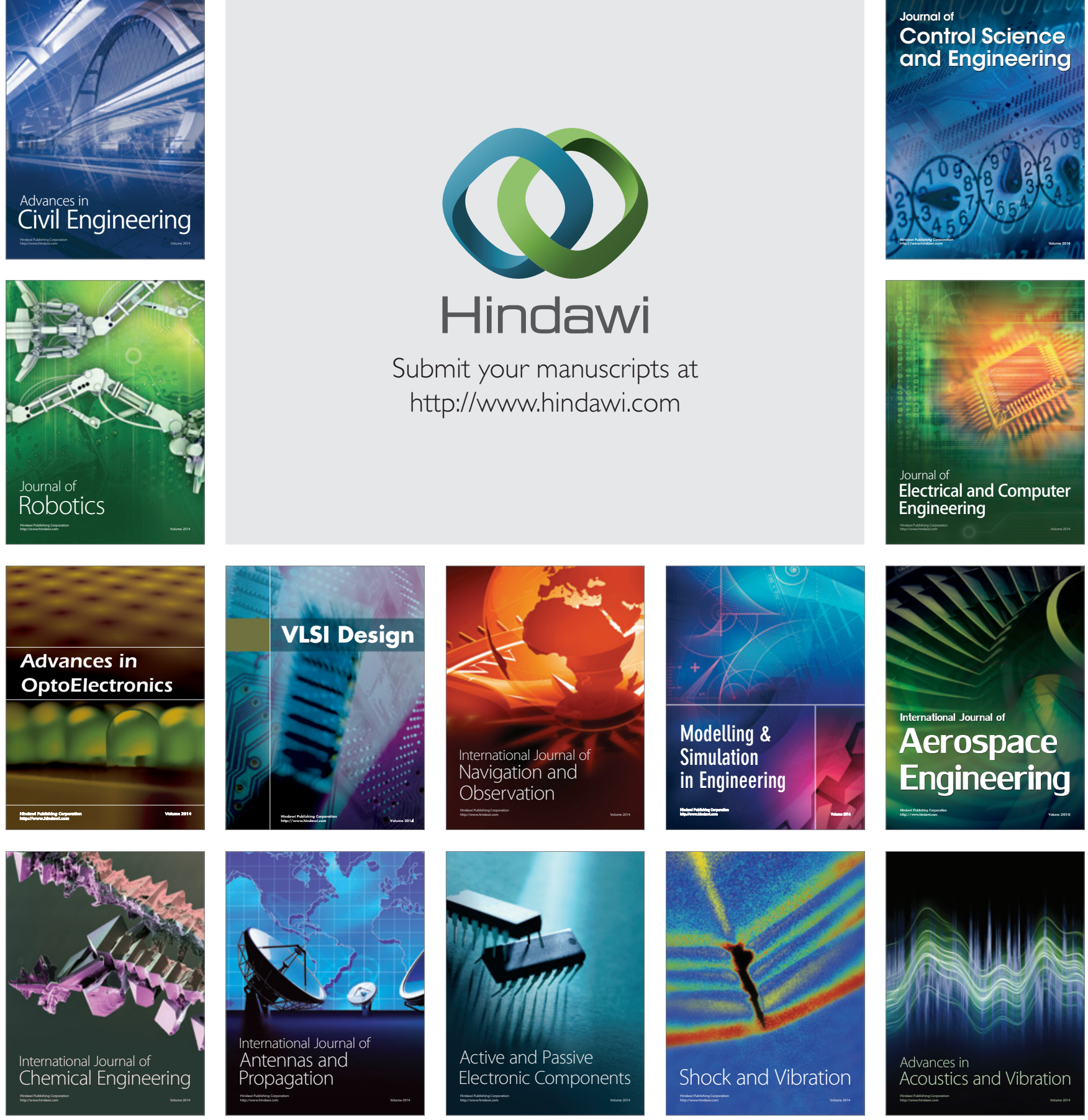